\title{
EMERGENT UNIVERSE WITH EXOTIC MATTER IN LOOP QUANTUM COSMOLOGY, DGP BRANE WORLD AND KALUZA-KLEIN COSMOLOGY
}

\author{
PRABIR RUDRA 1 \\ Department of Mathematics, Bengal Engineering and Science University, Shibpur, Howrah-711 103, India.
}

\begin{abstract}
In this work we have investigated the emergent scenario of the universe described by Loop quantum cosmology model, DGP brane model and Kaluza-Klein cosmology. Scalar field along with barotropic fluid as normal matter is considered as the matter content of the universe. In Loop quantum cosmology it is found that the emergent scenario is realized with the imposition of some conditions on the value of the density of normal matter in case of normal and phantom scalar field. This is a surprising result indeed considering the fact that scalar field is the dominating matter component! In case of Tachyonic field, emergent scenario is realized with some constraints on the value of $\rho_{1}$ for both normal and phantom tachyon. In case of DGP brane-world realization of an emergent scenario is possible almost unconditionally for normal and phantom fields. Plots and table have been generated to testify this fact. In case of tachyonic field emergent scenario is realized with some constraints on $\dot{H}$. In Kaluza-Klein cosmology emergent scenario is possible only for a closed universe in case of normal and phantom scalar field. For a tachyonic field realization of emergent universe is possible for all models(closed, open and flat).
\end{abstract}

\section{INTRODUCTION}

In the standard cosmological model, the existence of a big bang singularity in the early universe is still an open problem. In order to resolve this problem, Ellis et al. proposed, in the context of general relativity, a scenario, called an emergent universe [1, 2]. In this scenario, the space curvature is positive and the Universe stays, past eternally, in an Einstein static state and then evolves to a subsequent inflationary phase. Inflation is basically future eternal, which means that once inflation has started most of the volume of the universe will remain in an inflating state. Probably this is the possible mechanism responsible for the present cosmic acceleration [3, 4]. So, the Universe originates from an Einstein static state, rather than from a big bang singularity. It is also worth noting that an Einstein static state as the initial state of the Universe is also favored by the entropy considerations [5, 6 .

The inflationary universe emerges from a small static state that has within it the seeds for the development of the microscopic universe and it is called Emergent Universe scenario. The universe has a finite initial size, with a finite amount of inflation occurring over an infinite time in the past and with inflation then coming to an end via reheating in the standard way. References [7, 8, summarized the salient features of emergent universe as follows:

1. The universe is almost static at the finite past $(t \rightarrow-\infty)$ and isotropic, homogeneous at large scales;

2. It is ever existing and there is no time-like singularity;

3. The universe is always large enough so that the classical description of space-time is adequate;

4. The universe may contain exotic matter so that the energy conditions may be violated;

\footnotetext{
${ }^{1}$ prudra.math@gmail.com
} 
5. The universe is accelerating as suggested by recent measurements of distances of high redshift type Ia supernovae.

Ellis et al 2 provided a realization of a singularity-free inflationary universe in the form of a simple cosmological model dominated at early times by a single minimally coupled scalar field with a physically based potential. Mukherjee et al 7 presented a general framework for an emergent universe scenario and showed that emergent universe scenarios are not isolated solutions and they may occur for different combinations of radiation and matter. Campo et al 9] studied the emergent universe model in the context of a self-interacting Jordan-Brans-Dicke theory and showed that the model presents a stable past eternal static solution which eventually enters a phase where the stability of this solution is broken leading to an inflationary period. Debnath [8] discussed the behaviour of different stages of the evolution of the emergent universe considering that the universe is filled with normal matter and a phantom field. Mukherji and Chakraborty [10] developed EinsteinGauss-Bonnet (EGB) theory in the emergent universe scenario. They have considered the Friedman-LematreRobertson-Walker cosmological model in Horava gravity and the emergent scenario for all values of the spatial curvature. Paul et al 11] predicted the range of the permissible values for the parameters associated with the constraints on exotic matter needed for an emergent universe. The emergent universe in Horava gravity was studied by Mukherji and Chakraborty [12.

In recent years, loop Quantum Gravity (LQG) is an outstanding effort to describe the quantum effect of our universe 13, 14. LQG is a theory trying to quantize the gravity with a non-perturbative and background independent method. The theory and principles of LQG when applied in the cosmological framework creates a new theoretical framework of Loop Quantum Cosmology(LQC) 15, 16, 17. In this theory, classical spacetime continuum is replaced by a discrete quantum geometry. The effect of LQG can be described by the modification of Friedmann equation to add a term quadratic in density. In LQC, the non-perturbative effects lead to correction term $\frac{\rho_{T}^{2}}{\rho_{1}}$ to the standard Friedmann equation. With the inclusion of this term, the universe bounces quantum mechanically as the matter energy density reaches the level of $\rho_{1}$ (order of Plank density).

A simple and effective model of brane-gravity is the Dvali-Gabadadze-Porrati (DGP) braneworld model [41] which models our 4-dimensional world as a FRW brane embedded in a 5-dimensional Minkowski bulk. It explains the origin of dark energy $(\mathrm{DE})$ as the gravity on the brane leaking to the bulk at large scale. On the 4-dimensional brane the action of gravity is proportional to $M_{p}^{2}$ whereas in the bulk it is proportional to the corresponding quantity in 5-dimensions. The model is then characterized by a cross over length scale $r_{c}=\frac{M_{p}^{2}}{2 M_{5}^{2}}$ such that gravity is 4-dimensional theory at scales $a<<r_{c}$ where matter behaves as pressureless dust, but gravity leaks out into the bulk at scales $a>>r_{c}$ and matter approaches the behaviour of a cosmological constant. Moreover it has been shown that the standard Friedmann cosmology can be firmly embedded in DGP brane.

A fundamental theory on higher dimensional model was introduced by Kaluza-Klein [18, 19, where they considered an extra dimension with FRW metric to unify Maxwells theory of electromagnetism and Einsteins gravity. After extensive research, scientists realized that higher dimensional Cosmology may be more useful to understand the interaction of particles. Moreover, as our space-time is explicitly four dimensional in nature, the hidden dimensions must be related to the dark matter and dark energy. In modern physics Kaluza-Klein theory showed its great importance in string theory [20, in supergravity 21] and in superstring theories 22. Li et al in 23] have considered the inflation in Kaluza-Klein theory. In 1997, Overduin and Wesson [24] represented a review of Kaluza-Klein theory with higher dimensional unified theories. String cloud and domain walls with quark matter in N-dimensional Kaluza-Klein Cosmological model was presented by Adhav et al 25. Recently some authors [26, 27, 28, 29, 30, 31, 32] studied Kaluza-Klein cosmological models with different dark energies and dark matters. In this paper we want to investigate the possibilities for the realization of emergent universe for the above three models.

The general outline of the procedure followed in the paper is as follows: At first we fix $H$ as a function of the energy density and (possibly) pressure (this comes from the type of gravity we choose); then given the Lagrangian of the field one knows the energy density and pressure as functions of the field; finally, we choose the cosmology to be of emergent type, which fixes $\mathrm{H}$ (or the scale factor a) as function of time. The paper 
is organized as follows: Section 2 deals with the emergent scenario in Loop quantum cosmology. In section 3 we investigate the possibilities for emergent scenario in DGP brane-world model. In section 4 we discuss the emergent scenario for an universe described by Kaluza-Klein cosmology. Finally the paper ends with some concluding remarks in section 5 .

\section{EMERGENT SCENARIO IN LOOP QUANTUM COSMOLOGY}

Modified Friedmann equation for LQC is given by [33, 34, 35]

$$
H^{2}=\frac{\rho}{3}\left(1-\frac{\rho}{\rho_{1}}\right)
$$

Here $\rho_{1}=\sqrt{3} \pi^{2} \gamma^{3} G^{2} \hbar$ is the critical loop quantum density and $\gamma$ is the dimensionless Barbero-Immirzi parameter. The modified Raychaudhuri equation is given by,

$$
\dot{H}=-\frac{1}{2}(\rho+p)\left(1-2 \frac{\rho}{\rho_{1}}\right)
$$

where $\rho=\rho_{\phi}+\rho_{m}$, is the total energy density comprised of the energy density of normal matter, $\rho_{m}$ and energy density of exotic matter, $\rho_{\phi}$, in the form of scalar field.

In this work we consider exotic matter in the form of phantom field or tachyonic field and examine the possibility of an emergent universe. Normal matter in the form of barotropic fluid is considered to complement the exotic matter in a non-interacting scenario. The pressure and energy density of normal matter is connected by the relation,

$$
p_{m}=w \rho_{m}, \quad-1 \leq w \leq 1
$$

Now we consider that there is no interaction between normal matter and phantom field (or tachyonic field), so the normal matter and phantom field (or tachyonic field) are separately conserved. The energy conservation equations for normal matter and phantom field (or tachyonic field) are

$$
\dot{\rho_{m}}+3 H\left(\rho_{m}+p_{m}\right)=0
$$

and

$$
\dot{\rho_{\phi}}+3 H\left(\rho_{\phi}+p_{\phi}\right)=0
$$

From the eqn. (4) we get the expression for energy density of matter as

$$
\rho_{m}=\rho_{0} a^{-3(+w)}
$$

where $\rho_{0}$ is the integration constant.

For emergent model of the universe, the scale factor may be chosen as 7,8 ,

$$
a=a_{0}\left(\beta+e^{\alpha t}\right)^{n}
$$

where $a_{0}, \alpha, \beta$ and $n$ are positive constants.

The constant parameters are restricted as follows [12]:

1. $a_{0}>0$ for the scale factor a to be positive,

2. $\beta>0$, to avoid any singularity at finite time (big-rip)

3. $\alpha>0$ or $n>0$ for expanding model of the universe,

4. $\alpha<0$ and $n<0$ implies big bang singularity at $t=-\infty$. 
Therefore Hubble parameter and its derivatives are given by,

$$
H=\frac{n \alpha e^{\alpha t}}{\left(\beta+e^{\alpha t}\right)}, \quad \dot{H}=\frac{n \beta \alpha^{2} e^{\alpha t}}{\left(\beta+e^{\alpha t}\right)^{2}}, \quad \ddot{H}=\frac{n \beta \alpha^{3} e^{\alpha t}\left(\beta-e^{\alpha t}\right)}{\left(\beta+e^{\alpha t}\right)^{3}}
$$

From the above expressions it is quite clear that $H$ and $\dot{H}$ are both positive, but it is seen that $\ddot{H}$ changes $\operatorname{sign}$ at $t=\frac{1}{\alpha} \log \beta$. We see that $H, \dot{H}$ and $\ddot{H}$ all tend to zero as $t \rightarrow-\infty$. On the other hand as $t \rightarrow \infty$, the solution gives asymptotically a de Sitter universe. From the above choice of scale factor, the deceleration parameter $q$ can be simplified to the form,

$$
q=-1-\frac{\beta}{n e^{\alpha t}}
$$

\subsection{NORMAL OR PHANTOM FIELD:}

The kinetic energy term for phantom field has a negative sign. As a result of this, the ratio between the pressure and energy density is always less than -1 . The explicit form of energy density and pressure are as follows, 36.

$$
\begin{aligned}
\rho_{\phi} & =\frac{\delta}{2} \dot{\phi}^{2}+V(\phi) \\
p_{\phi} & =\frac{\delta}{2} \dot{\phi}^{2}-V(\phi)
\end{aligned}
$$

where $\delta=+1$ corresponds to the normal scalar field and $\delta=-1$ corresponds to the phantom scalar field. Now using equations (2), (10) and (11) we have,

$$
\dot{H}=-\frac{1}{2 \rho_{1}}\left[\delta \dot{\phi}^{2}+(1+w) \rho_{m}\right]\left[\rho_{1}-\delta \dot{\phi}^{2}-2 V(\phi)-2 \rho_{m}\right]
$$

This is a quadratic in $\dot{\phi}^{2}$ and we get,

$$
\dot{\phi}^{2}=\frac{-B+\sqrt{B^{2}-4 A C}}{2 A}
$$

where $A=\frac{\delta^{2}}{2 \rho_{1}}, B=\frac{\delta}{2 \rho_{1}}\left[(w+3) \rho_{m}-\rho_{1}+2 V(\phi)\right]$ and $C=-\left[\frac{\rho_{m}}{2 \rho_{1}}\left\{(1+w)\left(\rho_{1}-2 V(\phi)\right)-2\right\}-\dot{H}\right]$.

\subsubsection{Case I : For Normal scalar field}

For normal scalar field, $\delta=+1$. Therefore $B=\frac{1}{2 \rho_{1}}\left[(w+3) \rho_{m}-\rho_{1}+2 V(\phi)\right]$.

We have two cases:

1) for $B<0$ : We have $V(\phi)<\frac{1}{2}\left\{\rho_{1}-(w+3) \rho_{m}\right\}$. Now we should have $B^{2}-4 A C>0$. Since $A>0$, then $C<0$. Therefore we have $\dot{H}<\frac{\rho_{m}}{2 \rho_{1}}\left\{(1+w)\left(\rho_{1}-2 V(\phi)\right)-2\right\}$. Now in order to realize a universe undergoing accelerated expansion we should have $\dot{H}>0$. This is possible if $\rho_{1}-2 V(\phi)-2>0$, i.e., $V(\phi)<\frac{1}{2}\left(\rho_{1}-2\right)$. Now comparing this with the accepted range of $V(\phi)$, we get $w<\left(\frac{2}{\rho_{m}}-3\right)<1$, which implies $\rho_{m}>\frac{1}{2}$. This is a very interesting result.

2) for $B>0$ : We have $V(\phi)>\frac{1}{2}\left\{\rho_{1}-(w+3) \rho_{m}\right\}$. Now in order to make the R.H.S. of equation (13) positive definite, we should have $C<0$, which again leads us to the result $V(\phi)<\frac{1}{2}\left(\rho_{1}-2\right)$ for the accelerating universe just like the previous case. Now if we compare this with the accepted range for $V(\phi)$, we get a range for the values of the potential, $\frac{1}{2}\left\{\rho_{1}-(w+3) \rho_{m}\right\}<V(\phi)<\frac{1}{2}\left(\rho_{1}-2\right)$. From this we further get $w>\left(\frac{2}{\rho_{m}}-3\right)>-1$, which implies $\rho_{m}<1$. This is also a very important result. 


\subsubsection{Case II : For Phantom field}

For phantom field, $\delta=-1$. Therefore $B=-\frac{1}{2 \rho_{1}}\left[(w+3) \rho_{m}-\rho_{1}+2 V(\phi)\right]$.

Like before we have two cases:

1) for $B<0$ : We have $V(\phi)>\frac{1}{2}\left\{\rho_{1}-(w+3) \rho_{m}\right\}$. Now we should have $B^{2}-4 A C>0$. Hence this is just like sub-case 2 of normal scalar field. We get a range for the potential $V(\phi), \frac{1}{2}\left\{\rho_{1}-(w+3) \rho_{m}\right\}<V(\phi)<\frac{1}{2}\left(\rho_{1}-2\right)$. From this we further get $w>\left(\frac{2}{\rho_{m}}-3\right)>-1$, which implies $\rho_{m}<1$ for the accelerating universe.

2) for $B>0$ : We have $V(\phi)<\frac{1}{2}\left\{\rho_{1}-(w+3) \rho_{m}\right\}$. Now in order to make the R.H.S. of equation (13) positive definite, we should have $C<0$, i.e., $\dot{H}>0$. This again leads to sub-case 1 of the normal scalar field. Hence we should have $\rho_{1}-2 V(\phi)-2>0$, i.e., $V(\phi)<\frac{1}{2}\left(\rho_{1}-2\right)$ for the accelerating universe. Now comparing this with the accepted range of $V(\phi)$, we get $w<\left(\frac{2}{\rho_{m}}-3\right)<1$, which implies $\rho_{m}>\frac{1}{2}$.

\subsection{TACHYONIC FIELD}

The Lagrangian for the tachyonic field $\psi$ having potential $U(\psi)$ is given by [37, 38, 39, 40,

$$
L=-U(\psi) \sqrt{1-\epsilon \dot{\psi}^{2}}
$$

where $\epsilon=+1$ represents the normal tachyon and $\epsilon=-1$ represents phantom tachyon. The expressions for energy density and pressure are,

$$
\rho_{\psi}=\frac{U(\psi)}{\sqrt{1-\epsilon \dot{\psi}^{2}}}
$$

and,

$$
p_{\psi}=-U(\psi) \sqrt{1-\epsilon \dot{\psi}^{2}}
$$

Due to the above complicated forms of $\rho$ and $p$, it is not possible to find an expression for $\dot{\psi}^{2}$. However using the field equation for LQC, i.e., equation (2), we get,

$$
\dot{\psi}^{2}=\frac{2 \rho_{m}(1+w)\left(\rho_{\psi}+\rho_{m}\right)-\rho_{1} \rho_{m}(1+w)-2 \dot{H} \rho_{1}}{\rho_{1} \rho_{\psi} \epsilon-2 \epsilon \rho_{\psi}\left(\rho_{\psi}+\rho_{m}\right)}
$$

We know that $\dot{\psi}^{2}$ has to be positive. It means that both numerator and denominator are of the same sign.

\section{Case I: When both numerator and denominator are positive:}

For phantom tachyon, $\epsilon=-1$. We get $\rho_{1}<2 \rho$ and $\rho_{1}<2 \rho\left[\frac{\rho_{m}(1+w)}{\rho_{m}(1+w)+2 \dot{H}}\right]$. We know that for an accelerating universe $\dot{H}>0$. So keeping this in mind we combine the above two inequalities into a single one $\rho_{1}<2 \rho\left[\frac{\rho_{m}(1+w)}{\rho_{m}(1+w)+2 H}\right]$, i.e., $\rho_{1} \in\left(0,2 \rho\left[\frac{\rho_{m}(1+w)}{\rho_{m}(1+w)+2 H}\right]\right)$ since the second one implies the first.

For normal phantom, $\epsilon=1$. Here we get $\rho_{1}>2 \rho$ and $\rho_{1}<2 \rho\left[\frac{\rho_{m}(1+w)}{\rho_{m}(1+w)+2 H}\right]$. It is quite clear that for an accelerating universe the acceptable range of values for $\rho_{1}$ is $\rho_{1} \in\left(0,2 \rho\left[\frac{\rho_{m}(1+w)}{\rho_{m}(1+w)+2 H}\right]\right) \cup(2 \rho, \infty)$ 


\section{Case II: When both numerator and denominator are negative:}

For phantom tachyon, we get $\rho_{1}>2 \rho$ and $\rho_{1}>2 \rho\left[\frac{\rho_{m}(1+w)}{\rho_{m}(1+w)+2 H}\right]$. Combining the two inequalities for the accelerating universe we get the acceptable range of $\rho_{1}$ as $\rho_{1}>2 \rho$, i.e., $\rho_{1} \in(2 \rho, \infty)$

For normal tachyon we get $\rho_{1}<2 \rho$ and $\rho_{1}>2 \rho\left[\frac{\rho_{m}(1+w)}{\rho_{m}(1+w)+2 H}\right]$. So combining the above two we get the acceptable range for $\rho_{1}$ as $2 \rho\left[\frac{\rho_{m}(1+w)}{\rho_{m}(1+w)+2 H}\right]<\rho_{1}<2 \rho$, i.e., $\rho_{1} \in\left(2 \rho\left[\frac{\rho_{m}(1+w)}{\rho_{m}(1+w)+2 \dot{H}}\right], 2 \rho\right)$

\section{EMERGENT SCENARIO IN DGP BRANE-WORLD}

While flat, homogeneous and isotropic brane is being considered, the Friedmann equation in DGP brane model [41, 42, 43] is modified to the equation

$$
H^{2}=\left(\sqrt{\frac{\rho}{3}+\frac{1}{4 r_{c}^{2}}}+\epsilon^{\prime} \frac{1}{2 r_{c}}\right)^{2},
$$

where $H=\frac{\dot{a}}{a}$ is the Hubble parameter, $\rho$ is the total cosmic fluid energy density and $r_{c}=\frac{M_{p}^{2}}{2 M_{5}^{2}}$ is the cross-over scale which determines the transition from $4 \mathrm{D}$ to $5 \mathrm{D}$ behaviour and $\epsilon^{\prime}= \pm 1$ (choosing $M_{p}^{2}=8 \pi G=1$ ). For $\epsilon^{\prime}=+1$, we have standard DGP $(+)$ model which is self accelerating model without any form of DE, and effective $w$ is always non-phantom. However for $\epsilon^{\prime}=-1$, we have $\operatorname{DGP}(-)$ model which does not self accelerate but requires DE on the brane. It experiences $5 \mathrm{D}$ gravitational modifications to its dynamics which effectively screen DE. The modified Raychaudhuri's equation is given by

$$
\left(2 H-\frac{\epsilon^{\prime}}{r_{c}}\right) \dot{H}=-H(\rho+p)
$$

\subsection{NORMAL OR PHANTOM FIELD:}

The relevant expressions for $\rho$ and $p$ are given by equations (10) and (11) respectively. Now using equations (18), (19) and using the values of $\rho$ and $p$ from equations (10) and (11) we get,

$$
\begin{gathered}
\frac{1}{r}\left[\left\{\rho_{m}(w+1)+\delta \dot{\phi}^{2}\right\}^{2}\left\{3\left(1+\epsilon^{\prime 2}\right)+2 r_{c}^{2}\left(2 V(\phi)+2 \rho_{m}+\delta \dot{\phi}^{2}\right)+2 r_{c} \sqrt{3} \epsilon^{\prime} \sqrt{\frac{3}{r_{c}^{2}}+4 V(\phi)+4 \rho_{m}+2 \delta \dot{\phi}^{2}}\right\}\right. \\
\left.-4\left\{3+2 r_{c}^{2}\left(2 V(\phi)+2 \rho_{m}+\delta \dot{\phi}^{2}\right)\right\} \dot{H}^{2}\right]=0
\end{gathered}
$$

This is a 8 degree equation in $\dot{\phi}$. It is not possible to find an explicit expression for $\dot{\phi}$ in terms of the given parameters. So we intend to find a numerical solution of the above equation by considering different sets of values of the parameters involved. The following plots and the table show the possible outcomes of the procedure undertaken and the positive solutions of $\dot{\phi}$ obtained for different sets of values of the parameters.

From the plots below it can be seen that on most occasions we get a positive root of $\dot{\phi}$ from equation (20), irrespective of whatever sets of values we choose for the associated parameters. Both the branches of DGP brane model has been taken into account and both normal and phantom fields have been considered in the plots. In some cases there are some constraints specially for low values of $n$, very high values of $r_{c}$ and extremely low values of $V(\phi)$. In these cases positive roots are difficult to achieve, and hence the realization of the emergent scenario. Other than these trivial cases we can easily experience the emergent universe for DGP brane model in normal and phantom scalar field associated with barotropic fluid as normal matter. 


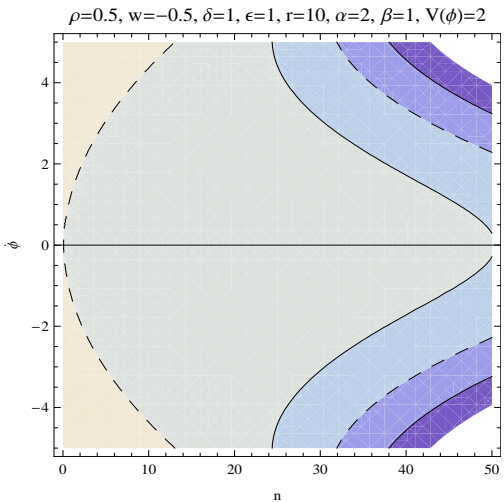

Fig. 1

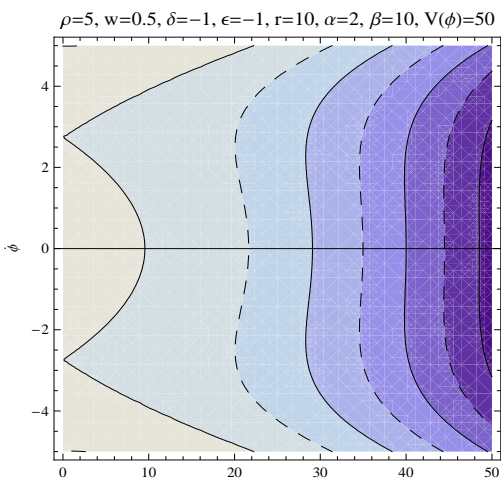

Fig. 4

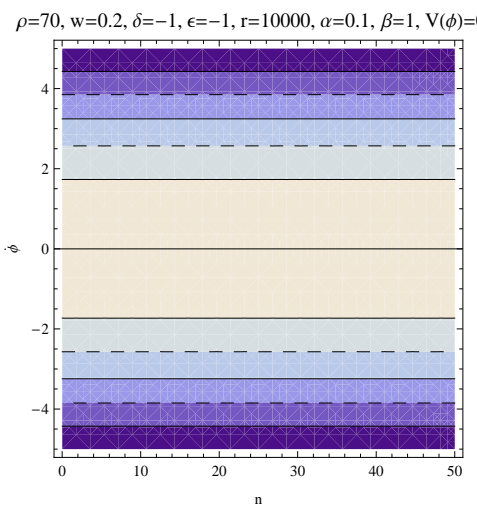

Fig. 7

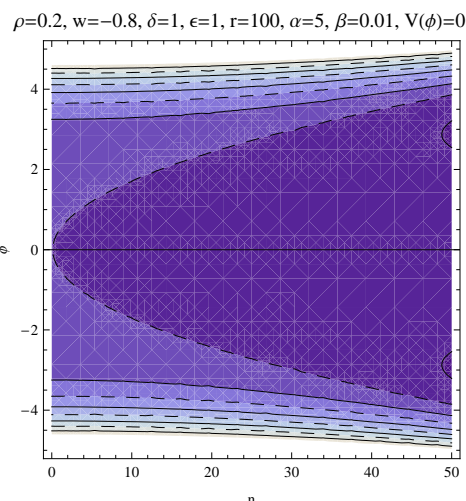

Fig. 2

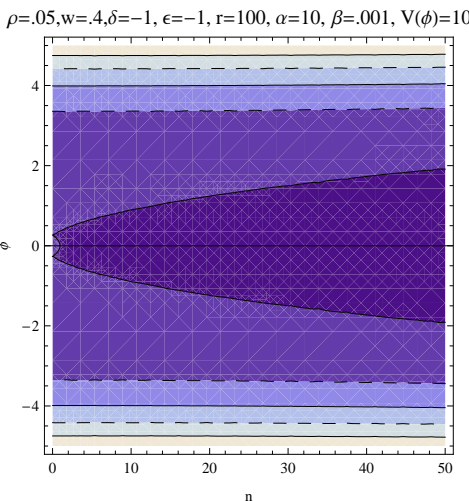

Fig. 5

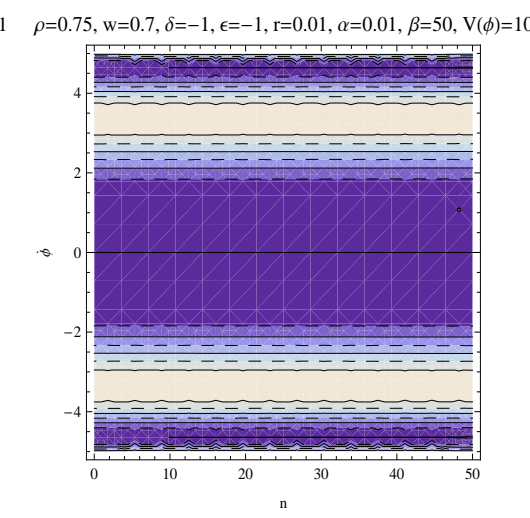

Fig. 8

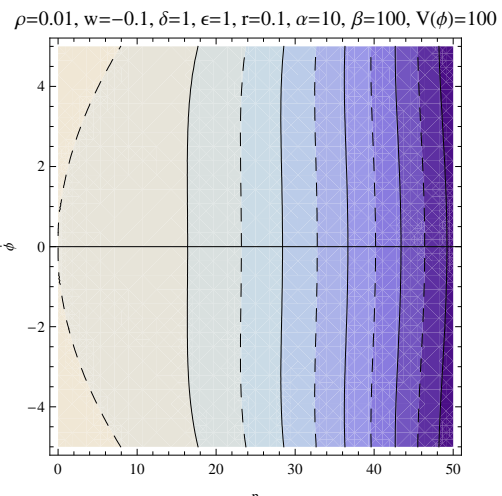

Fig. 3

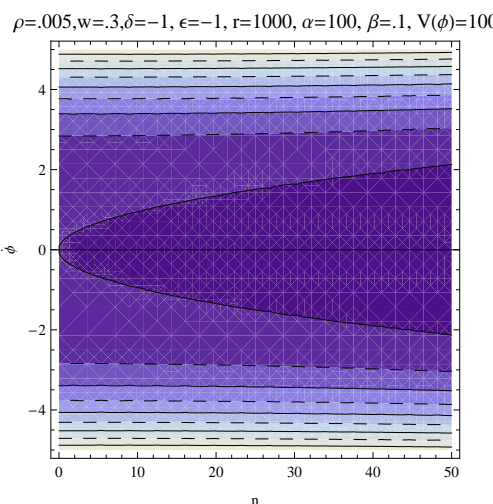

Fig. 6 


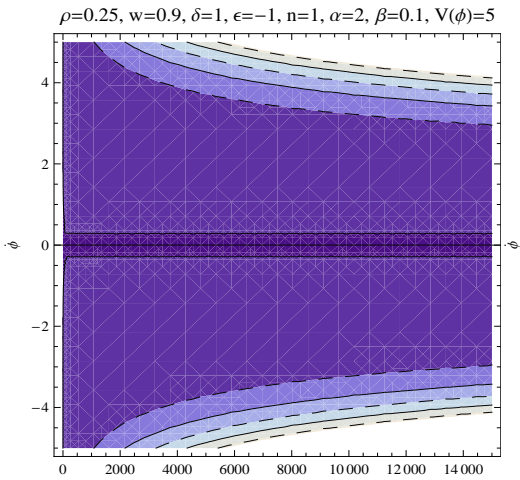

Fig. 9

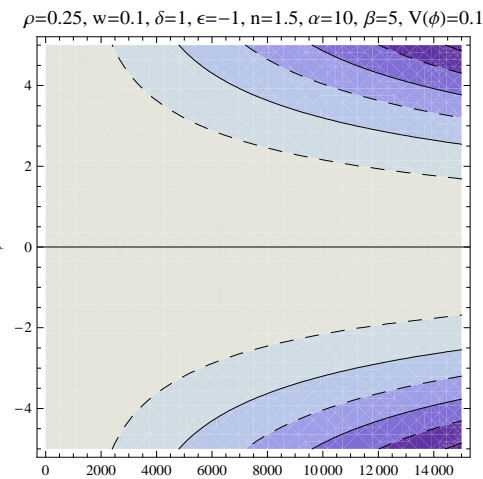

Fig. 10

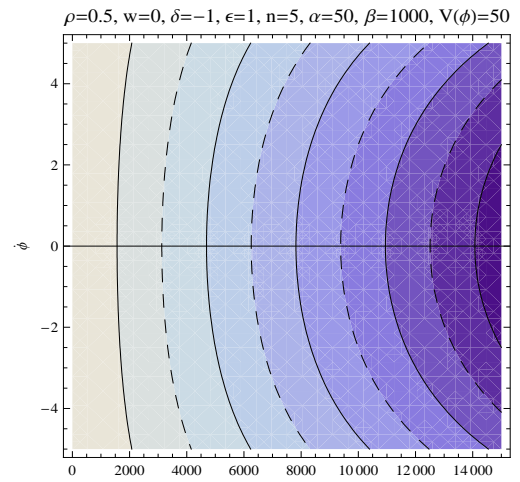

Fig. 11

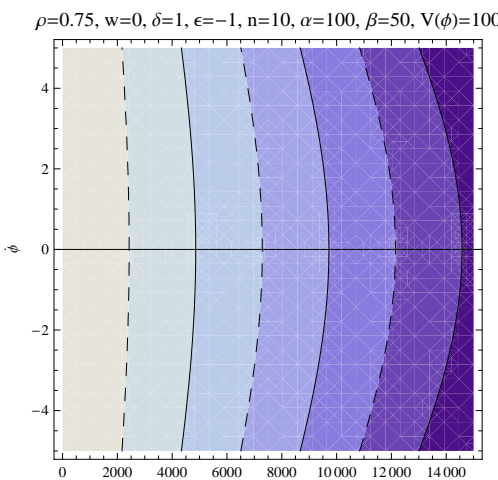

Fig. 12

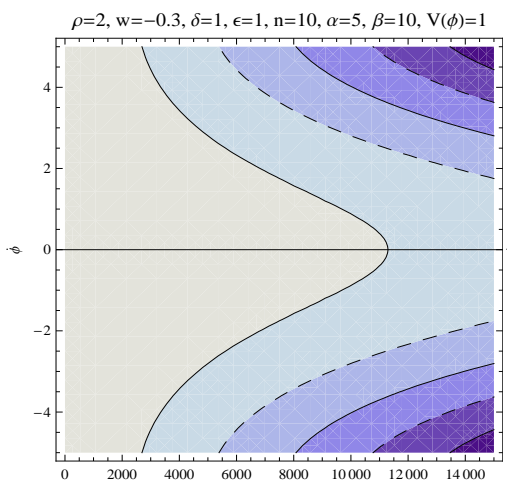

Fig. 15

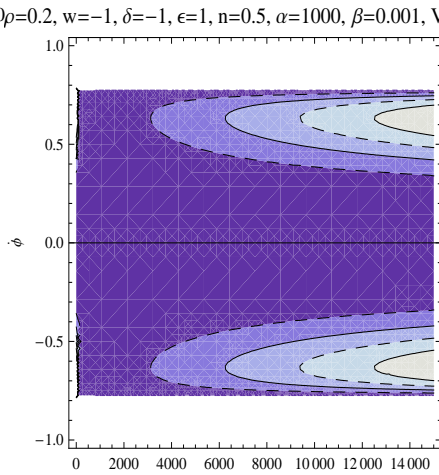

Fig. 13

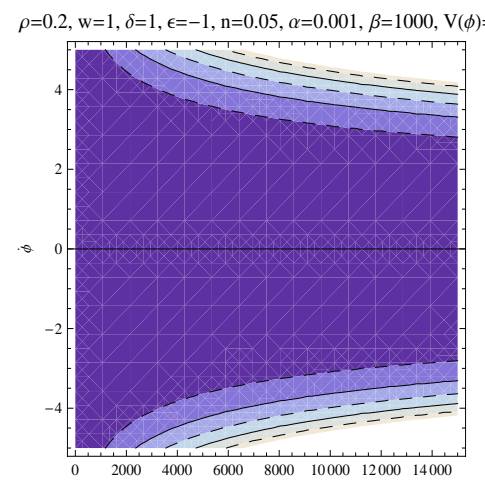

Fig. 14

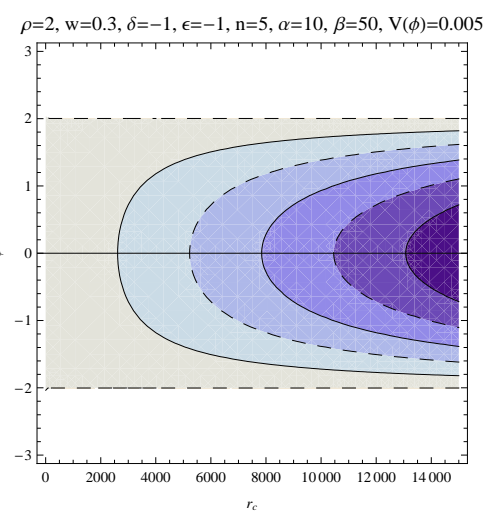

Fig. 16

The above figures represent the plot of $\dot{\phi}$ against $r_{c}$ (in equation (20)) with the variation of different parameters. The different values of the parameters chosen have been provided with the plots. 


\begin{tabular}{|c|c|c|c|c|c|c|c|c|c|}
\hline$\rho_{m}$ & $w$ & $\delta$ & $\epsilon^{\prime}$ & $r_{c}$ & $n$ & $\alpha$ & $\beta$ & $V(\phi)$ & Positive roots $(\dot{\phi})$ \\
\hline 0.5 & -0.5 & 1 & 1 & 10 & 0.1 & 2 & 1 & 2 & - \\
\hline 0.2 & -0.8 & 1 & 1 & 100 & 0.7 & 5 & 0.01 & 0.01 & 0.407962 \\
\hline 0.01 & -0.1 & 1 & 1 & 0.1 & 1 & 10 & 100 & 100 & 1.76366 \\
\hline 5 & 0.5 & -1 & -1 & 10 & 1.2 & 2 & 10 & 50 & 2.90562 \\
\hline 0.05 & 0.4 & -1 & -1 & 100 & 1.5 & 10 & 0.001 & 1000 & 0.424633 \\
\hline 0.005 & 0.3 & -1 & -1 & 1000 & 2 & 100 & 0.1 & 100 & 14.1425 \\
\hline 70 & 0.2 & -1 & -1 & 10000 & 10 & 0.1 & 100 & 0.1 & - \\
\hline 0.75 & 0.7 & -1 & -1 & 0.01 & 100 & 0.01 & 50 & 10 & 4.57297 \\
\hline 0.25 & 0.9 & 1 & -1 & 1 & 1 & 2 & 0.1 & 5 & 0.61914 \\
\hline 0.25 & 0.1 & 1 & -1 & 10 & 1.5 & 10 & 5 & 0.1 & 8.31793 \\
\hline 0.5 & 0 & -1 & 1 & 100 & 5 & 50 & 100 & 50 & 10.0499 \\
\hline 0.75 & 0 & 1 & -1 & 1000 & 10 & 100 & 50 & 100 & 21.2419 \\
\hline 0.2 & -1 & -1 & 1 & 0.2 & 0.5 & 1000 & 0.001 & 0.1 & $4.32136 \times 10^{-21}$ \\
\hline 0.2 & 1 & 1 & -1 & 0.2 & 0.05 & 0.001 & 1000 & 2000 & - \\
\hline 2 & -0.3 & 1 & 1 & 0.5 & 10 & 5 & 10 & 1 & 6.66419 \\
\hline 2 & 0.3 & -1 & -1 & 10 & 5 & 10 & 50 & 0.005 & 2.00624 \\
\hline
\end{tabular}

Table I: Nature of the roots $(\dot{\phi})$ of the equation (20) for various values of parameters involved.

Above we have prepared a table showing the positive roots of $\dot{\phi}$ for different sets of values of the parameters. Both self-accelerating $\left(\epsilon^{\prime}=1\right)$ and non-self accelerating $\left(\epsilon^{\prime}=-1\right)$ branches of DGP brane model has been considered. Moreover both Normal scalar field $(\delta=1)$ and phantom scalar $(\delta=-1)$ have been considered while finding the roots. From the table it is seen that in general there are no positive roots of $\dot{\phi}$ for very small value of $n$. For very high value of $r_{c}$ as well we are deprived of a positive root. Hence in these cases there is some difficulty in realizing the emergent scenario. So it can be concluded that $n$ and $r_{c}$ are the two really sensitive quantities that govern the realization of an emergent scenario in the DGP brane model. The dependence on $r_{c}$ is quite obvious, since it is the cross over scale from $4 D$ to $5 D$. A very large value of $r_{c}$ definitely alters the basic nature of the model and may be responsible for any sort of adverse phenomenon. But the dependence on $n$ is not quite clear and remains an intriguing question. Perhaps its appearance in the numerator of the expression of Hubble parameter and its derivatives, gives it enough independence to play a major role in determining the fate of the universe. But barring these special cases we are definitely able to realize the emergent scenario for DGP brane model on a general basis. 


\subsection{TACHYONIC FIELD}

Using the field equation for DGP brane, i.e., equations (18) and (19) with the equations (15) and (16) we get,

$$
\dot{\psi}^{2}=\frac{\rho_{m}(1+w)\left(\sqrt{\frac{4 r_{c}^{2} \rho+3}{3}}+\epsilon^{\prime}\right)-2 \dot{H} \sqrt{\frac{4 r_{c}^{2} \rho+3}{3}}}{\left(\sqrt{\frac{4 r_{c}^{2} \rho+3}{3}}+\epsilon^{\prime}\right) \rho_{\psi} \epsilon}
$$

In order to make the R.H.S. of the above equation positive definite we should have,

$$
\dot{H}<\frac{\rho_{m}(1+w)\left(\alpha+\epsilon^{\prime}\right)}{2 \alpha}
$$

where $\alpha=\sqrt{\frac{4 r_{c}^{2} \rho+3}{3}}$. The above relation gives the condition for the emergent universe with Tachyonic field in DGP brane model. Now in order to be consistent with the recent cosmic acceleration the above relation for the emergent universe gives the following range for $\dot{H}, \dot{H} \in\left(0, \frac{\rho_{m}(1+w)\left(\alpha+\epsilon^{\prime}\right)}{2 \alpha}\right)$.

\section{EMERGENT SCENARIO IN KALUZA-KLEIN COSMOLOGY}

Einstein's field equation for Kaluza-Klein space-time is given by,

$$
H^{2}+\frac{k}{a^{2}}=\frac{4 \pi G}{3} \rho
$$

and

$$
\dot{H}+2 H^{2}+\frac{k}{a^{2}}=-\frac{8 \pi G}{3} p
$$

\subsection{NORMAL OR PHANTOM FIELD:}

Using equations (23) and (24) we have,

$$
\dot{H}-\frac{k}{a^{2}}=-\frac{8 \pi G}{3}(p+\rho)
$$

Substituting the values of $\rho$ and $p$ from equations (3), (10) and (11) in the above equation we get,

$$
\frac{8 \pi G}{3}\left\{\delta \dot{\phi}^{2}+\rho_{m}(1+w)\right\}+\left(\dot{H}-\frac{k}{a^{2}}\right)=0
$$

From the above equation, we get,

$$
\dot{\phi}^{2}=\frac{1}{\delta}\left[\frac{3\left(k-a^{2} \dot{H}\right)}{8 a^{2} \pi G}-\rho_{m}(1+w)\right]
$$

\subsubsection{Case-I: For Normal Scalar field}

For this case $\delta=1$. Hence putting this in equation (27) we get,

$$
\dot{\phi}^{2}=\frac{3\left(k-a^{2} \dot{H}\right)}{8 \pi G a^{2}}-\rho_{m}(1+w)
$$


From the above equation we see that the L.H.S is non-negative. Hence in order that the R.H.S. is also nonnegative we should have

$$
\rho_{m}<\frac{3\left(k-a^{2} \dot{H}\right)}{8 \pi G a^{2}(1+w)}
$$

Depending on the above value, we discuss the possibilities of all the three types of universe.

\section{Subcase - I : For Closed Universe, $(\mathbf{k}>0)$}

In equation (29), since the L.H.S. is positive, hence we should have $\dot{H}<\frac{k}{a^{2}}$. Since $k$ is positive we can realize an emergent scenario in the range $\dot{H} \in\left(0, \frac{k}{a^{2}}\right)$.

\section{Subcase - II : For Open Universe, $(\mathbf{k}<\mathbf{0})$}

For this case we see that since $k<0$, we should have $\dot{H}<0$ in order to make the R.H.S of equation (29) positive. But this result is contrary to cosmic acceleration, which is unacceptable.

\section{Subcase - III : For Flat Universe, $(k=0)$}

In this case $k=0$. Using this in equation (29), we get $\dot{H}<0$, just like the previous case. Hence this case is also un-physical.

So the only physically acceptable situation is the first case, which is the closed universe.

\subsubsection{Case-II: For Phantom field}

For this case $\delta=-1$. Hence putting this in equation (27) we get,

$$
\dot{\phi}^{2}=\rho_{m}(1+w)-\frac{3\left(k-a^{2} \dot{H}\right)}{8 \pi G a^{2}}
$$

Here in order to make the R.H.S. positive definite, we should have

$$
\rho_{m}>\frac{3\left(k-a^{2} \dot{H}\right)}{8 \pi G a^{2}(1+w)}
$$

We discuss the different possibilities below:

\section{Subcase - I : For Closed Universe, $(k>0)$}

In this case $\dot{H} \leq \frac{k}{a^{2}} \Rightarrow \dot{H}>0$ in the range $\dot{H} \in\left(0, \frac{k}{a^{2}}\right)$, since $k>0$. Hence we can realize the emergent scenario in this range, consistent with the cosmic acceleration.

\section{Subcase - II : For Open Universe, $(\mathbf{k}<\mathbf{0})$}

Just like the Normal scalar field we do not realize the emergent universe in this case.

$$
\text { Subcase - III : For Flat Universe, }(k=0)
$$


In this case, like the normal scalar field we get $\dot{H}<0$. Hence this case is also un-physical and the emergent scenario is out of question.

Hence here also the emergent scenario is realized only for closed universe.

\subsection{TACHYONIC FIELD}

Using equations (16) and (24), we get,

$$
\left[\frac{\frac{3}{8 \pi G}\left(\dot{H}+2 H^{2}+\frac{k}{a^{2}}\right)-w \rho_{m}}{U(\psi)}\right]^{2}=1-\epsilon \dot{\psi}^{2}
$$

L.H.S. of the above equation is positive definite, and this is possible for all values of $k$, i.e., for open, closed and flat model of the universe. So it is possible to have emergent scenario for any model of universe(open, closed or flat) with Tachyonic field as matter content.

\section{CONCLUSION}

In this paper we have investigated the conditions that help to realize the emergent scenario of the universe. Three different models of universe were considered for the discussion, namely, Loop quantum cosmology, DGP brane-world and Kaluza-Klein cosmology. Normal, Phantom and Tachyonic fields have been considered separately for each model. In Tachyonic field, both normal tachyon and phantom tachyon has been considered for our evaluation process. To complement the scalar fields barotropic fluid has been chosen as the normal matter content. In case of Loop quantum cosmology the emergent scenario is realized with a restriction on the value of the density of normal matter, $\rho_{m}$ for normal and phantom field, which is really an unexpected result as the dominating matter component is the scalar field. For Tachyonic field emergent scenario is realized with constraints on the value of $\rho_{1}$ for both normal and phantom tachyon which is quite understandable. It is found that the realization of emergent scenario in case of DGP brane model is possible almost unconditionally in most cases for normal and phantom scalar fields except in a few exceptional cases. Plots were generated in order to confirm this. A table containing useful data has also been provided. In case of tachyonic field we get a constraint on $\dot{H}$. In case of Kaluza-Klein cosmology emergent scenario is realized only for closed universe in case of normal and phantom scalar fields. Finally with tachyonic field as matter content emergent scenario is realized for all the models(closed, open, flat) of the universe irrespective of any conditions.

\section{References}

[1] Ellis, G. F. R., Maartens, R. Class. Quant. Grav. 21, 223 (2004).

[2] Ellis, G. F. R., Murugan, J., Tsagas, C. G. Class. Quant. Grav. 21, 233 (2004).

[3] Perlmutter, S. et al. :- [Supernova Cosmology Project Collaboration], ApJ 517, 565(1999) [arXiv:astro-ph/9812133].

[4] Spergel, D. N. et al. :- WMAP Collaboration,Astron. J. Suppl 148, 175(2003) [arXiv astro-ph/0302209].

[5] Gibbons, G. W. :- Nucl. Phys. B 292, 784 (1987);

[6] Gibbons, G. W. :- Nucl. Phys. B 310, 636 (1988).

[7] Mukherjee, S., Paul, B. C., Dadhich, N., K., Maharaj, S. D., Beesham, A. : Class. Quantum. Grav. 23, $6927(2006)$. 
[8] Debnath, U. : Class. Quantum. Grav. 25, 205019(2008).

[9] S. del Campo et al (2007) JCAP 11030.

[10] S. Mukerji and S. Chakraborty (2010), Int. J. Theor. Phys. 492446.

[11] B. C. Paul et al(2010), Mon. Not. R. Astron. Soc. 407, 415419.

[12] Mukerji, S., Chakraborty, S. Astrophys. Space Sc. DOI: 10.1007/s10509-010-0456-1 (2010)

[13] Rovelli, C. :- liv. Rev. Rel. 11(1998)

[14] Ashtekar, A., Lewandowski, J. :- Class. Quantum. Grav. 21R53(2004)

[15] Ashtekar, A. :- AIP Conf. Proc. 8613(2006)

[16] Bojowald, M. :- liv. Rev. Rel. 811(2005)

[17] Ashtekar, A. et al :- Adv. Theor. Math. Phys. 7233(2003)

[18] Kaluza, T. :- Preus. Acad. Wiss F1 9669(1921)

[19] Klein, O. :- A. Phys 37895 (1926)

[20] Polchinski, J. :- String Theory, Vols. I and II (Cambridge Univ. Press, 1998).

[21] Duff, M. J., Nilsson, B. E. W., Pope, C. N. :- Phys. Rep. 1301 (1986).

[22] Green, M. B., Schwarz, J. H., Witten, E. :- Superstring Theory (Cambridge Univ. Press, 1987).

[23] Li, L. X., Gott, I., Richard, J. :- Phys. Rev. D 58103513 (1998).

[24] Overduin, J. M., Wesson, P., S. :- Phys. Rep. 283303 (1997).

[25] Adhav, K. S., Nimkar, A. S., Dawande, M. V., :- Int. J. Theor. Phys. 472002 (2008).

[26] Qiang, L. E., Ma, Y. G., Han, M. X., Yu, D. :- Phys. Rev. D 71061501 (2005).

[27] Chen, S., Jing, J. :- J. Cosmol. Astropart. Phys. 09 001(2009).

[28] Ponce de Leon, J. :- Gen. Relativ. Gravit. 20539 (1988).

[29] Chi, L. K. :- Gen. Relativ. Gravit. 221347 (1990).

[30] Fukui, T. :- Gen. Relativ. Gravit. 25931 (1993).

[31] Liu, H., Wesson, P. S. :- Int. J. Mod. Phys. D 3627 (1994).

[32] Coley, A. A. :- Astrophys. J. 427585 (1994).

[33] Wu, P., Zhang, S.N. : J. Cosmol. Astropart. Phys. 06, 007 (2008)

[34] Chen, S., Wang, B., Jing, J. : Phys. Rev. D 78, 123503 (2008)

[35] Fu, X., Yu, H., Wu, P. : Phys. Rev. D 78, 063001 (2008)

[36] Chang, B., Liu, H., Xu, L., Zhang, C. : Chin. Phys. Lett. 24 2153(2007) [arXiv:0704.3768(astro-ph)]

[37] Hao, J. G., Li, X. -z. : Phys. Rev. D 68, 043510 (2003)

[38] Hao, J. G., Li, X. -z. : Phys. Rev. D 68, 083514 (2003)

[39] Nojiri, S., Odintsov, S. D. : Phys. Lett. B 571,1 (2003)

[40] Gumjudpai, B., Naskar, T., Sami, M., Tsujikawa, S., : JCAP 0506007 (2005)

[41] Dvali, G. R., Gabadadze, G., Porrati, M.:- Phys.Lett. B 485 208(2000)[arXiv:000506(hep-th)].

[42] Deffayet, D.:- Phys.Lett. B 502 199(2001).

[43] Deffayet, D., Dvali, G.R., Gabadadze, G.:-Phys.Rev.D 65044023 (2002)[arXiv:0105068(astro-ph)]. 